\title{
Directional Writing Dependence of Birefringence in Multicomponent Silica-based Glasses with Ultrashort Laser Irradiation
}

\author{
Chaxing FAN ${ }^{* 1,2}$, Bertrand POUMELLEC ${ }^{* 2}$, Huidan ZENG $^{* 1,2}$, Matthieu LANCRY ${ }^{* 2}$, Weijia YANG ${ }^{* 2}$, Bernard \\ BOURGUIGNON $^{* 3}$ and Guorong CHEN ${ }^{* 1}$ \\ ${ }^{* 1}$ Key Laboratory for Ultrafine Materials of Ministry of Education, School of Materials Science and Engineering, East \\ China University of Science and Technology, Shanghai 200237, China \\ E-mail: Bertrand.Poumellec@u-psud.fr \\ *2 Institut de Chimie Moléculaire et des Matériaux d'Orsay (ICMMO), UMR CNRS-UPS 8182, and \\ *3 Institut des Sciences Moléculaires d'Orsay, CNRS and Université de Paris Sud 11, Bâtiment 350, 91405 Orsay, France.
}

\begin{abstract}
We reveal laser-induced high birefringence in multicomponent silica-based glass by means of ultrashort laser irradiation. It is attributed to the residual stress. A systematic study of birefringence variation is carried out according to laser parameters such as pulse energy, writing speed and direction, and laser polarization. Strong writing directional dependence is clearly observed when the writing direction is perpendicular to the laser polarization at the writing speeds of $20 \mu \mathrm{m} / \mathrm{s}-120$ $\mu \mathrm{m} / \mathrm{s}$, which was likely due to an asymmetry of the laser beam or an asymmetry of interaction mechanism.
\end{abstract}

DOI:10.2961/jlmn.2011.02.0011

Keywords: birefringence, ultrashort laser, stress, $\mathrm{LiNbO}_{3}$ glass, directional writing dependence

\section{Introduction}

The use of femtosecond laser to rationally manipulate micro-/nano-structures inside transparent materials arises a myriad of material interactions, such as surface machining, annealing, 3D refractive index changes profiling, and simultaneously provides enormous potentialities in the development of monolithic micro-optics or composite electro-optic devices. Recently, other properties have also arisen, like chiral mechanical structures [1], directional dependent writing [1-4], oxidation-reduction [5] and cluster modification [6] so on. To better understand the femtosecond laser-matter interaction and exploit more opportunities in manipulation of 3D multi-functional photonic devices, it is thus obvious that a characterization of the response to the femtosecond irradiation of the key materials already developed and used in photonics applications is anticipated. Depending on the laser intensity and numerical aperture of microscope objective, refractive index changes in silica glass can be defined into three regimes with two "damage" thresholds [3], the first damage threshold T1 (e.g. $0.10 \mu \mathrm{J} /$ pulse in pure silica, $800 \mathrm{~nm}, 160$ fs, $100 \mathrm{kHz}, 0.55 \mathrm{NA}$ ) being the appearance of isotropic index change in the plan perpendicular to the direction of light propagation and the second damage threshold T2 (e.g. $0.31 \mu \mathrm{J} /$ pulse in pure silica, $800 \mathrm{~nm}, 160 \mathrm{fs}, 100 \mathrm{kHz}, 0.55$ $\mathrm{NA}$, for the polarization parallel to the writing direction) being the appearance of form birefringence in the plan perpendicular to the light propagation axis. The index change decays within a few seconds as the pulse energy below $\mathrm{T} 1$ while a permanent isotropic index change appears above T1 and below T2. Above T2, the index change is highly anisotropic and a strong linear birefringence is observed, which is mainly due to the appearance of nanogratings [7]. To our knowledge, birefringence has been observed in pure silica or slightly doped silica e.g. Ge-doped, F-doped silica and P-doped silica [8] but also in multicomponent glasses that contain noble metal nanoparticles. It is related in this case with particle deformation. However, in BK7 (another kind of multicomponent glass), no such property is observed [9], although strong damage occurs. We show in this paper that it is also possible to obtain birefringence in a multicomponent glass. We expected to obtain it originating from non-centrosymmetric lithium niobate nanocrystals induced by ultrashort laser irradiation in $32.5 \mathrm{Li}_{2} \mathrm{O}$ $27.5 \mathrm{Nb}_{2} \mathrm{O}_{5}-40 \mathrm{SiO}_{2}$ (LNS) glasses but it seems not.

Nevertheless, we present controllable experimental conditions for inducing strong birefringence in an homogeneous LNS glass by means of ultrashort laser irradiation. In order to open the possibility to repeat the same effects as in pure silica, a systematic study of birefringence variation was investigated according to the laser parameters such as pulse energy, writing speed, writing direction and laser polarization.

\section{Experimental section}

\subsection{Preparation of LNS glass}

The molar composition of LNS glass studied in this paper was $32.5 \mathrm{Li}_{2} \mathrm{O}-27.5 \mathrm{Nb}_{2} \mathrm{O}_{5}-40 \mathrm{SiO}_{2}$. Approximately 50 $\mathrm{g}$ batches of $\mathrm{Li}_{2} \mathrm{CO}_{3}, \mathrm{Nb}_{2} \mathrm{O}_{5}$ and $\mathrm{SiO}_{2}$ raw materials were mixed and melted in $\mathrm{Pt}$ crucible in an electronic furnace at $1430{ }^{\circ} \mathrm{C}$ for $2 \mathrm{hr}$. Then the melt was poured onto a steel plate heat-treated at $500{ }^{\circ} \mathrm{C}$, and transferred to another electronic furnace pre-set at $540{ }^{\circ} \mathrm{C}$ cooling down to room temperature. The transparent glass was annealed to release the stress at the decreasing rate of $20^{\circ} \mathrm{C} / \mathrm{hr}$ for the first $6 \mathrm{hr}$. After that, the glass samples were cut and mirror-polished with the size of $2 \mathrm{~mm} \times 10 \mathrm{~mm} \times 10 \mathrm{~mm}$, which were used for the irradiation experiments.

\subsection{Irradiation experiments}


The irradiation experimental procedure is already described in Ref. [2] precisely. We recall here only a few details. The laser radiation, in a Gaussian mode, produced by a regenerative amplified mode-locked Ti: Sapphire laser 120 fs pulse duration, $1 \mathrm{kHz}$, operating at $800 \mathrm{~nm}$. Considering that the propagation vector $\vec{k}$ is along 庉 direction, the beam was focused $180 \mu \mathrm{m}$ below the entry surface by $20 \mathrm{x}$ microscope objective $(\mathrm{NA}=0.6)$. The sample was moved along a direction perpendicular (let us say $\vec{x}$ ) to the laser beam either in one direction or in the other, thereby tracing continuous lines. We need to make a difference, in this paper, about direction and orientation of a line. We consider that a line in space has one orientation which can be scanned in two directions. The polarization lied along $y$, i.e. perpendicular to (perpendicular configuration) or parallel to (parallel configuration) the sample displacement. The laser beam pulse energy and the writing speed were varied from 0.12 up to $6.6 \mu \mathrm{J} /$ pulse and from $120 \mu \mathrm{m} / \mathrm{s}$ down to $1 \mu \mathrm{m} / \mathrm{s}$, respectively. The spacing between lines was $50 \mu \mathrm{m}$. For closer distance, there is an overlap of the photo-induced effects between neighboring lines.

After writing the lines, the sample was cleaved in the $y, z$ plan. The topography of the cleaved faces (crossing the written lines) was then observed using a phase shift interferometric microscope (PSI) and scanning electron microscope (SEM).

\subsection{Quantitative birefringence measurements}

For the method of Sénarmont compensator, we utilized an Olympus BX60 optical microscope equipped with a fixed quarter-wave plate $(\lambda / 4$ plate) and a rotatable analyzer to measure the amount of retardation from a specimen, which is proportional to birefringence. Since $\lambda / 4$ plates are designed for use at a specific wavelength, microscope illumination must be monochromatic, typically at the 546 $\mathrm{nm}$ green light. When the specimen is positioned diagonally between two crossed polarizer, the combined action of the specimen plus the $\lambda / 4$ plate generates linearly polarized waves whose $\mathrm{E}$ vectors are tilted at some azimuth $\alpha$ depending on the amount of retardation from the specimen. To measure the amount of retardation from this specimen, firstly the slow axis of the specimen is oriented at $45^{\circ}$ with respect to the crossed polarizer and analyzer, and then the fixed $\lambda / 4$-plate inserted in the microscope in order that the large axis of its wavefront ellipsoid is oriented parallel to the transmission axis of the analyzer. From the relative retardation $\psi$ by this method $\psi=2 \alpha$, we can calculate the birefringence of the specimen when its thickness is known.

$R=\frac{546}{180} \times \alpha$

where $\mathrm{R}$ is the retardance of the sample.

$B=\frac{R}{t}$

where $\mathrm{B}$ is the quantitative birefringence of the sample, and $t$ is the exact thickness of the region which produced the birefringence. In this work, we assumed that the thickness of the birefringent layer could be assimilated to the whole length of the laser tracks. In this manner, we computed a minimum of birefringence. Typically, the values measured by this method have a $\pm 0.3 \mathrm{~nm}$ error.

\section{Results}

\subsection{Varying pulse energy}

In relatively gentle irradiation conditions, strong dependence of birefringence in $\mathrm{x}$, $\mathrm{y}$ plane on pulse intensity was observed in LNS glass. We have checked that the neutral axis of the lines are oriented parallel and perpendicular to the writing direction of the line. Fig. 1a shows the image of the irradiated lines (two lines per group) taken in crossed polarizers, which were written at the same speed $(6 \mu \mathrm{m} / \mathrm{s})$ and focus depth. Pulse energy was decreased from the left to the right from $6.6 \mu \mathrm{J}$ to 0.12 $\mu \mathrm{J} /$ pulse in the case of writing direction perpendicular to the laser polarization. Obviously, the transmitted light intensity of the irradiated lines derived from birefringence gradually became weaker with the decrease of pulse energy. When the pulse energy was at $0.12 \mu \mathrm{J} /$ pulse, little transmitted light from the written lines positioned in the lower right corner of the image could be observed.
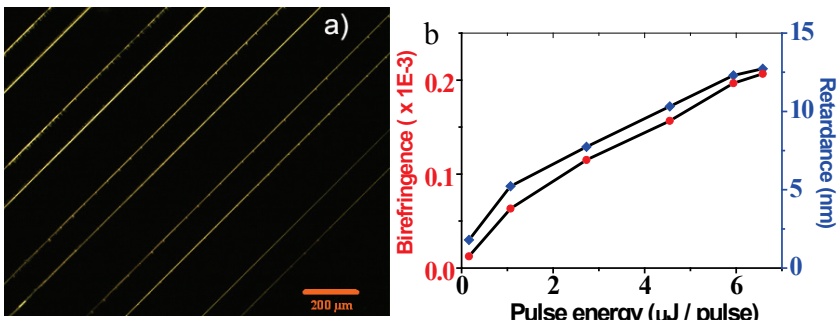

Fig. 1 a) Photograph image taken between crossed polarizers with the sample $45^{\circ}$ of the polarizer axis; b) Plot of birefringence (red dots) and retardance (blue dots) with respect to pulse energy. The pulse energy of each group (two lines in each group) is for $6.6 \mu \mathrm{J} /$ pulse, 5.9 $\mu \mathrm{J} /$ pulse, $4.6 \mu \mathrm{J} /$ pulse, $2.7 \mu \mathrm{J} /$ pulse, $1.1 \mu \mathrm{J} /$ pulse and $0.12 \mu \mathrm{J} /$ pulse from left to right. Writing speed $=6$ $\mu \mathrm{m} / \mathrm{s}$ and the polarization is perpendicular to writing orientation (perpendicular configuration).
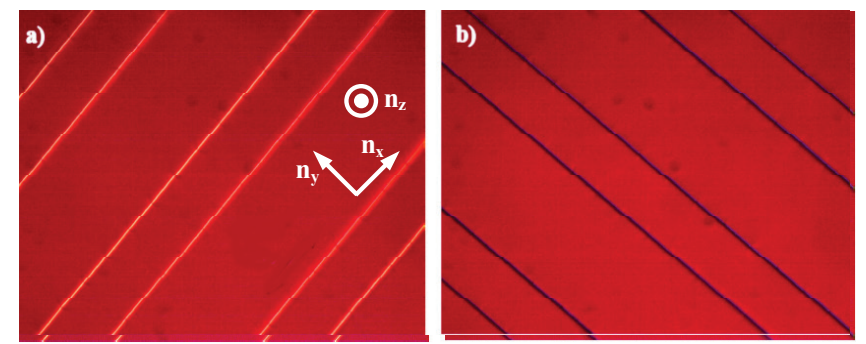

Fig. 2 a) Images taken between crossed polarizers and full wave plate with the sample $45^{\circ}$ and (b) $-45^{\circ}$ of the polarizer axis. The orientation of $\mathrm{x}, \mathrm{y}, \mathrm{z}$ directions are shown as inserted in a). The slow axis of full wave is north-east/south-west. Blue color indicates that slow axis of the material and the full wave are aligned.

We have determined the orientation of slow axis by means of a full wave $\lambda$ plate (the first order retardation plate) and find that it is always perpendicular to the lines. As depicted in Fig.2, yellow interference colors are 
observed when the sample is oriented parallel to the slow axis of the full wave plate, while blue colors result from rotating the sample by $90^{\circ}$. Thus, the birefringence in the $\mathrm{x}$, $\mathrm{y}$ plane deduced from the irradiated lines was $\mathrm{n}_{\mathrm{x}}<\mathrm{n}_{\mathrm{y}}$, and the slow axis of the birefringent region is oriented at $\sim 90^{\circ}$ from the written lines. Furthermore, the magnitude of birefringence measured by Sénarmont compensator was consistent with the above observation. The obtained birefringence was in the range of $10^{-4}-10^{-3}$ depending on the pulse energy, e.g. without optimization typ. $>2 \times 10^{-4}$ for a pulse energy of $6.6 \mu \mathrm{J}$ (Fig. 1b).

On the other hand, the modified region appears to be uniaxial because of the observation of the interference pattern using converging polarized light with a Bertrand lens.

\subsection{Varying writing speed}

Writing speed is another important factor of pulse intensity, which defines the overlap between two subsequent pulses. For instance, the overlap at $10 \mu \mathrm{m} / \mathrm{s}$ speed in this work is $99.5 \%$. It can be estimated by the formula,

Overlap $=1-\mathrm{v} /(\mathrm{f} * \mathrm{D})$,

where $f$ is the repetition rate, $v$ is the writing speed and $D$ is the laser beam diameter [3]. When the writing speed perpendicular to the laser polarization was varied from 1 to $10 \mu \mathrm{m} / \mathrm{s}$ at the pulse energy of $1.25 \mu \mathrm{J}$, the birefringence deduced from the irradiated lines decreased slight by $25 \%$. With higher writing speed, we have detected a directional dependence. The effect is strong and we were able to determine the birefringence from each writing direction quantitatively (Fig. 5a).

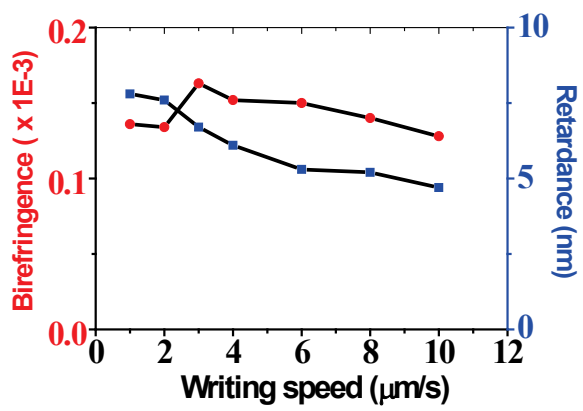

Fig. 3 Plot of birefringence (red dots) and retardance (blue dots) with respect to writing speed. Fixed pulse energy is $1.25 \mu \mathrm{J}$. (perpendicular configuration)

\subsection{Varying laser polarization}

Directional dependent photosensitivity and chiral structures in pure silica have early been demonstrated by our group when the femtosecond laser beam wrote lines along the $+x$ and $-x$ directions $[1,10]$. It was reported that the shearing in the head of the laser tracks changed with different writing directions (pen effect). Furthermore, it was found that the writing directional dependence was strongly related to the laser polarization. In this work, the same phenomena in LNS glass is observed when the writing speed was above $10 \mu \mathrm{m} / \mathrm{s}$.

Figure 4 depicts two examples of retardance laserinduced by different polarization orientations together with the writing speed at fixed pulse energy of $2.3 \mu \mathrm{J}$ in LNS glass. Firstly, we worked on perpendicular configuration and the writing direction was horizontal perpendicular to vertical polarization. We can observe that the transmitted light intensity derived from birefringence is at a different decreasing pace with the increase of writing speed between left and right directions (Fig. 4a, b). However, when we changed to parallel configuration and kept the same other parameters, no difference is detected between opposite directions (Fig. 4c, d).

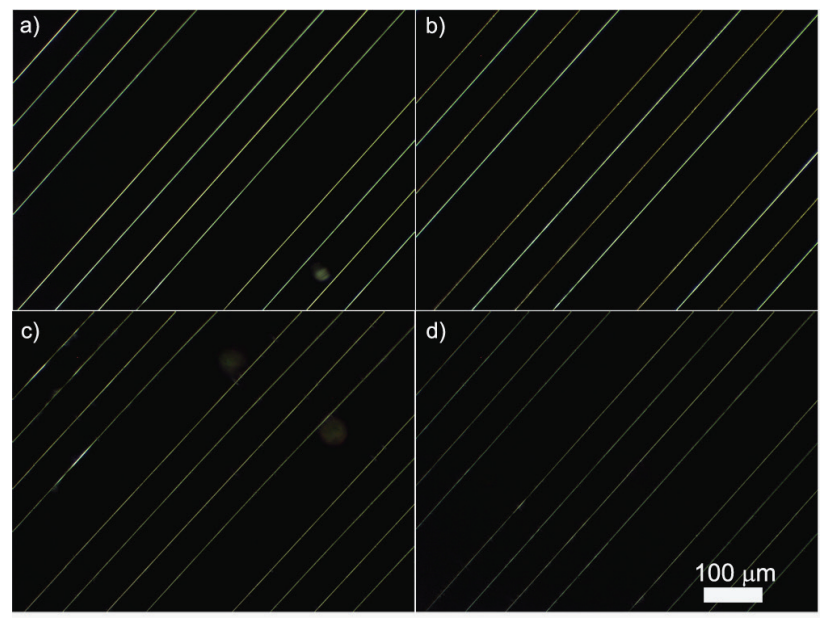

Fig. 4 Photograph images taken between crossed polarizers with rotation the sample $45^{\circ}$; a, b) At writing speeds of $10,20,40,80,100$ to $120 \mu \mathrm{m} / \mathrm{s}$ leftwards/rightwards in perpendicular configuration; c, d) At writing speeds of $10,20,40,80,100$ to $120 \mu \mathrm{m} / \mathrm{s}$ leftwards/rightwards in parallel configuration. (Four lines in each group)
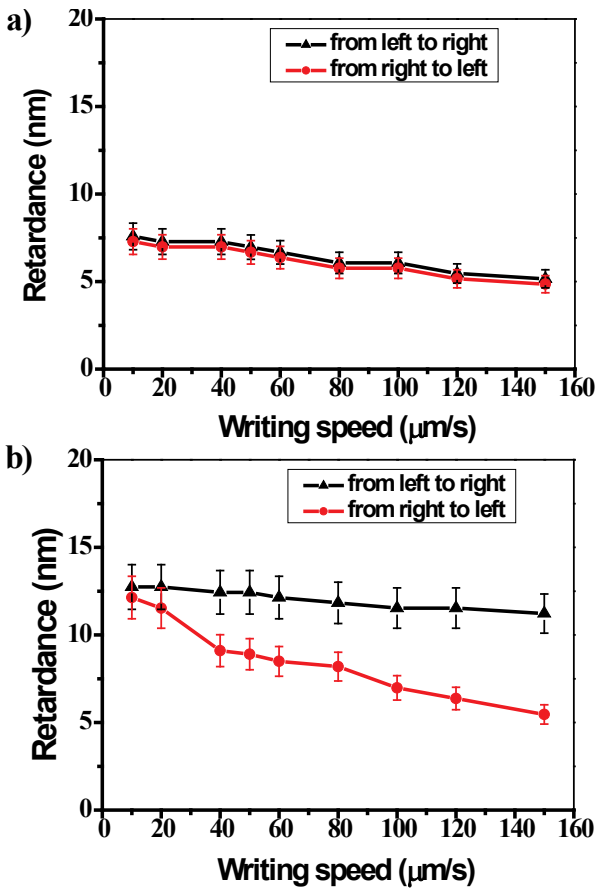

Fig. 5 Plot of the retardance with respect to writing speed in left-to-right direction (black dots) and right-to-left direction (red dots). a) perpendicular configuration; b) parallel configuration. Fixed pulse energy $=2.3$ $\mu \mathrm{J} /$ pulse. 
It should be noted that the perpendicular configuration here is different with the above cases, the polarization of which is horizontal and the displacement of the sample is along the left-to-right and right-to-left directions. The writing directional dependence in the case of vertical and horizontal orthogonal polarizations was significantly different. To confirm this phenomenon, Fig. 5a shows a noticeable retardance change due to the writing directional dependence according to the writing speed. The retardance difference between left-to-right and right-to-left directions is larger as the increase of the writing speed, which is opposite to the phenomenon observed in pure silica [3]. The retardance of the left-to-right direction at the speed of $120 \mu \mathrm{m} / \mathrm{s}$ is about $11.5 \mathrm{~nm}$, as many as roughly twice 6.4 $\mathrm{nm}$ of the right-to-left direction. However, with rotation the polarizer $90^{\circ}$ to orient the laser polarization parallel to the writing direction, no writing directional dependence was observed at speeds of 10 to $120 \mu \mathrm{m} / \mathrm{s}$ (Fig. 5b). Apparently, the retardance at each speed is also smaller than the one upon the perpendicular configuration.

\subsection{Laser track structure}

For better investigating the structures of laser tracks, we observed the cross section surface of the cleaved glass by two methods: PSI (before polishing and etching) and SEM (after polishing and etching).

\section{Surface topography}

Surface topography of cross section (YZ plane) of the cleaved glass in which four lines were previously written alternatively left-to-right and right-to-left, is shown in Fig. 6. The writing direction is perpendicular to the laser polarization, and other writing conditions are shown in the Figure caption.

Fig. 6 2D images of surface topography obtained by PSI with $\mathrm{x} 40$ objective in the (yz) plane. Cross sections of four laser tracks alternatively written left-to-right/right-toleft following $\mathrm{x}$ axis are shown. The femtosecond laser propagation axis is from bottom to top. Laser conditions: $800 \mathrm{~nm}, 120 \mathrm{fs}, 1 \mathrm{kHz}, \mathrm{NA}=0.6$, writing speed $=6 \mu \mathrm{m} / \mathrm{s}$. (a) $5.94 \mu \mathrm{J} /$ pulse; b) $4.55 \mu \mathrm{J} /$ pulse. Black regions are not measurable areas due to out of range, level or slope or strong scattering.

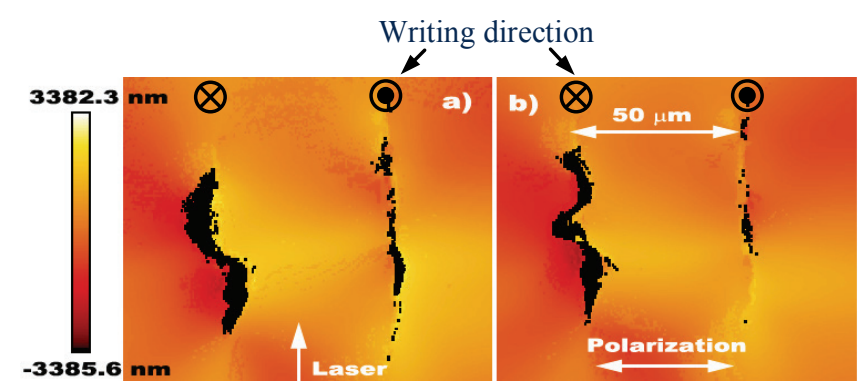

It is important to note that, 1) Large different topographies have been obtained between left-to-right and right-to-left directions, for which we observed asymmetric structures around the head and the tail of the laser tracks. A large discontinuity is detected in left-to-right direction by comparison with a small one for the other direction. In both cases, it originates from shear stress. The strain induced in opposite directions is different. 2) The topography appears to depend also on pulse energy. The different shapes of surface topography of the trace in the left-to-right direction presented a phase shift when the pulse energy was increased.

\section{SEM images}

We observed the cross section surface of the cleaved glass by virtue of SEM after polishing and etching it with $10 \%$ hydrofluoric (HF) acid for 5 mins. Fig. 7 shows SEM images of cross section of four laser tracks at different pulse energy. They are lens-shaped. The width of the laser trace is $\sim 3 \mu \mathrm{m}$ at maximum close to the diameter of laser beam. The lengths of laser tracks at 5.94 and $2.73 \mu \mathrm{J} /$ pulse are $\sim 57$ and $\sim 49 \mu \mathrm{m}$, respectively. The dark parts in Fig.7a was some holes resulting from the etching because of nonhomogeneous densification irradiated by femtosecond laser. We did not observe similar holes before the etching. It has been clearly revealed that self-organized nanogratings/nanocracks traces in pure silica is induced by intermediate intensity of femtosecond laser, resulting in form birefringence [7]. However, here when the laser intensity was increased from 0.12 up to $6.6 \mu \mathrm{J} /$ pulse, such nanostructures were not visible inside the laser traces in LNS glass (as insert in Fig. 7a.). Further measurements on the cross section of laser tracks were carried out by electron backscattered diffraction (EBSD). Non-crystalline area was detected by the absence of EBSD diffraction patterns. Hence, it can be concluded that the birefringence induced in this matter was not attributed to "form" birefringence or crystals but to the anisotropic residual stress.

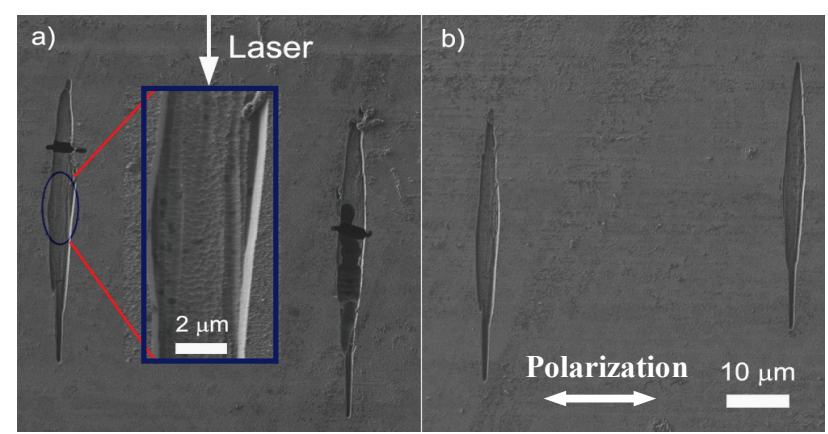


Fig. 7 SEM images of cross sections of four laser tracks alternatively written left-to-right/right-to-left following $\mathrm{x}$ axis after cleaved and etched with $10 \%$ HF for 5 mins. The femtosecond laser propagation axis is from bottom to top. The laser polarization was in the picture plan. Laser conditions: $800 \mathrm{~nm}, 120 \mathrm{fs}, 1 \mathrm{kHz}, \mathrm{NA}=0.6$, writing speed $=6 \mu \mathrm{m} / \mathrm{s}$. (a) $5.94 \mu \mathrm{J} /$ pulse; b) 2.73 $\mu \mathrm{J} /$ pulse. (Blue circled area was enlarged as insert)

\section{Discussion}

We find out a retardance of $13 \mathrm{~nm}$ without optimization typ. $>2 \times 10^{-4}$ for a pulse energy of $6.6 \mu \mathrm{J}$. This value is larger than the one we can deduce from typical stress fields. All these results were approximately produced from $\sim 50$ $\mu \mathrm{m}$ thickness of birefringent layer. It indicated that tens of magnitude of birefringence in this sample could be obtained by writing multilayers along the laser propagation. It was already demonstrated that the topography of surface relaxation of a cleaving of silica plates revealed the stress/strain fields induced by femtosecond laser, as we mentioned in Ref.[2]. Until now, modification of materials has been shown differently for laser writing in opposite directions in pure silica glass [1,2]. Shearing in the head of laser tracks was observed reversing under writing direction reversing. In LNS glass, it is considered that the structures imprinted was identically originated from plasma density structures [11], and the writing directional dependence was likely due to an asymmetry of the laser beam and /or an asymmetry of interaction mechanism.

For better understanding the writing directional dependence, the imprinting effect on the matter of asymmetric laser beam was schemed in Fig. 8. The intensity distribution of laser beam exhibits an up-down asymmetry according to the writing direction, which was represented by the color gradient of the row. It should be noted here that the laser processing is involved in multipulses overlapping. When the laser was writing in the leftto-right, the bottom of laser pulse was imprinted in the matter while the top of laser pulse would be imprinted in the right-to-left direction. Therefore, we can suggest that the writing directional dependence was likely resulted from an asymmetry of the laser beam.

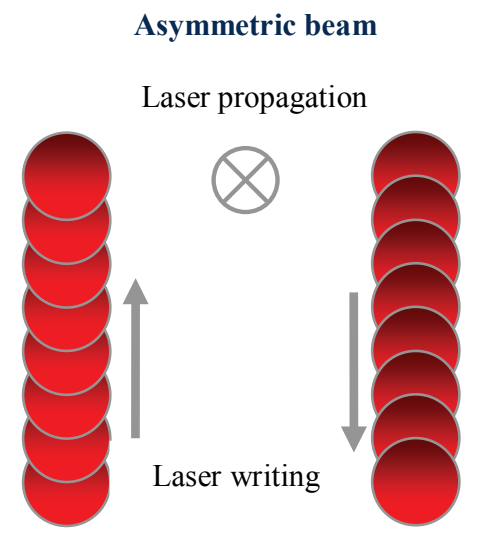

Fig. 8 Imprinting effect on the matter of asymmetric laser beam.

A noticeable polarization sensitivity is also observed. In particular, the effect is clearly different and it is not matter of just amplitude. In one configuration, there is a directional effect, with the other configuration, there is none. Therefore, even if polarization effect in plasma has been theorized in kinetic regime [12], the recording in the material is not yet explained at all.

Moreover, the writing speed affects the writing directional dependence largely but we observe an unexpected effect. It is commonly understandable that when overlap decreases when speed increases, the directional dependence decreases but here it is the reverse! We are currently working on this effect.

\section{Conclusion}

Femtosecond laser writing in LNS glass presents some unique photosensitivity exploiting more opportunities to fabricate multi-dimensional and optical-sensitive devices. We revealed that the photo-induced high birefringence was attributed to the residual stress accompanied with ultrashort laser irradiation, which was different with form birefringence resulted from sub-wavelength period structures in pure silica. It was demonstrated by observation of surface topography of a cleaved glass and high resolution SEM images of polished and etched laser traces, which were not observed nano-cracks/gratings. Laser-induced high birefringence originated from the residual stress was dependent on the pulse intensity, writing speed and direction, and the laser polarization. When the writing speed was in the range of $20 \mu \mathrm{m} / \mathrm{s}-120$ $\mu \mathrm{m} / \mathrm{s}$ with the direction perpendicular to the laser polarization, strong writing directional dependence was apparent. The directional dependence was elucidated by the overlapping mutilpulse due to an asymmetry of the laser beam and / or an asymmetry of interaction mechanism. Furthermore, when we remained the laser irradiation parameters except the polarization parallel to the writing direction, no writing directional dependence appeared. The mechanism of the polarization effect is presently under investigation, and the interaction of laser-matter in LNS glass is still in progress by increasing the laser repetition rate.

\section{Acknowledgments and Appendixes}

(1) French Embassy in Shanghai (Eiffel grant run by French Ministry of Foreign Affair) for the thesis financial supports.

(2) FP7-PEOPLE-International Incoming Fellowship (IIF) FemtoNano 219582

\section{References}

[1] B. Poumellec, M. Lancry, J. C. Poulin and S. Ani Joseph: Opt. Express, 16, (2008) 18354.

[2] B. Poumellec, L. Sudrie, M. Franco, B. Prade, A. Mysyrowicz: Opt. Express, 11, (2003) 1070.

[3] M. Lancry, W. Yang, B. Poumellec and Bernard Bourguignon, Femtosecond Laser Microfabrication, OSA Technical Digest (CD) (Optical Society of America, 2009), ISBN 978-1-55752-879-7 (2009).

[4] P. G. Kazansky, W. Yang, E. Bricchi, J. Bovatsek, A. Arai, Y. Shimotsuma, K. Miura and K. Hirao: Appl. Phys. Lett., 90, (2007) 151120. 
[5] J. Qiu, X. Jiang, C. Zhu, M. Shirai, J. Si, N. Jiang, K. Hirao: Angew. Chem. Int. Ed., 43, (2004) 2230.

[6] A. Akin Unal, A. Stalmashonak, G. Seifert, and H. Graener: Phys. Review B, 79, (2009) 115411.

[7] Y. Shimotsuma, P. G. Kazansky, J. R. Qiu, K. Hirao: Phys. Rev. Lett., 91, (2003) 247405-1.

[8] M. Lancry, W. Yang, B. Poumellec, and P. Kazansky, Advances in Optical Materials, OSA Technical Digest (CD) (Optical Society of America, 2009), ISBN 978-155752-879-7, paper AWB4 (2009).

[9] C. Hnatovsky, R. S. Taylor, E. Simova, P. P. Rajeev, D. M. Rayner, V. R. Bhardwaj, and P. B. Corkum: Apl. Phys. A 84 (2006) 47.

[10] P. G. Kazansky, Y. Shimotsuma: J. Ceram. Soc. Jpn., 16, (2008) 1052.

[11] A. Couairon, L. Sudrie, M. Franco, B. Prade, and A. Mysyrowicz: Phys. Rev. B 71, (2005) 125435.

[12] V V Belyi, Y. A Kukharenko: J. Phys.: Conference Series 35, (2006) 71-77.

(Received: June 07, 2010, Accepted: August 18, 2011) 